$$
\begin{array}{r}
\text { Pontifícia Universidade Católica } \\
\text { Do Rio de Janeiro }
\end{array}
$$

Pedro A. M-S. David

\title{
Formação do Preço, Atração de Investimentos e Gerenciamento de Risco no Mercado Brasileiro de Energia Elétrica
}

\begin{abstract}
Tese apresentada ao Programa de Pós-Graduação em Engenharia Elétrica da PUC-Rio como requisito parcial para obtenção do título de Doutor em Engenharia Elétrica.
\end{abstract}

\section{Professores Orientadores:}

Alvaro Veiga Filho, Docteur Mario Veiga Ferraz Pereira, D.Sc. Sergio Granville, Ph.D.

Rio de Janeiro Fevereiro de 2004 


$$
\begin{array}{r}
\text { Pontifícia Universidade Católica } \\
\text { DO Rio de Janeiro }
\end{array}
$$

Pedro Américo Moretz-Sohn David

Formação do Preço, Atração de Investimentos e Gerenciamento de Risco no Mercado Brasileiro de Energia Elétrica

Tese de Doutorado apresentada como requisito parcial para obtenção do grau de Doutor pelo Programa de PósGraduação em Engenharia Elétrica do Departamento de Engenharia Elétrica do Centro Técnico Científico da PUCRio. Aprovada pela Comissão Examinadora abaixo assinada.

\author{
Dr. Álvaro de Lima Veiga Filho \\ Orientador \\ Departamento de Engenharia Elétrica - PUC-Rio \\ Dr. Mário Veiga Ferraz Pereira \\ Co-Orientador \\ PSR Consultoria Ltda \\ Dr. Sérgio Granville \\ Co-Orientador \\ PSR Consultoria Ltda \\ Dr. Afonso Henriques M. Santos \\ UNIFEI \\ Dr. Edson Luiz da Silva \\ UFSC \\ Dra. Mônica Barros \\ Departamento de Engenharia Elétrica - PUC-Rio \\ Dr. Boris Garbati Gorenstin \\ FURNAS \\ Prof. José Eugenio Leal \\ Coordenador Setorial do Centro \\ Técnico Científico - PUC-Rio
}

Rio de Janeiro, 06 de fevereiro de 2004 
Todos os direitos reservados. É proibida a reprodução total ou parcial do trabalho sem autorização da universidade, do autor e do orientador.

\section{Pedro A. M-S. David}

Graduou-se em Engenharia Elétrica na EE-UFRJ em 1974 e pós graduou em Engenharia Elétrica na PUC-Rio, em 1995, na área de Controle Digital e Processamento de Sinais. Possui diversos cursos de especialização em Engenharia Elétrica e Informática e participou de diversos projetos e estudos, entre os quais destacam-se o projeto do Sistema de Transmissão em Corrente Contínua de Alta Tensão (CCAT) de Itaipu; estudos préoperativos de sistemas de alta e extra-alta tensão; estudos de viabilidade de expansão de geração e análises de risco-retorno no mercado brasileiro de energia elétrica.

Ficha Catalográfica

David, Pedro A. M-S.

Formação do preço, atração de investimentos e gerenciamento de risco no mercado brasileiro de energia elétrica / Pedro A. M-S. David; orientadores: Alvaro Veiga Filho, Mario Veiga Ferraz Pereira e Sergio Granville. - Rio de Janeiro: PUC, Departamento de Engenharia Elétrica, 2004.

134 f.; il.; $30 \mathrm{~cm}$

Tese (doutorado) - Pontifícia Universidade Católica do Rio de Janeiro, Departamento de Engenharia Elétrica.

Inclui referências bibliográficas.

1. Engenharia Elétrica - Teses. 2. Mercado de energia elétrica. 3. Modelos de mercado e formação do preço. 4. Viabilidade de investimentos em geração. 5. Derivativos no mercado de energia elétrica. 6. Aversão ao risco. I. Veiga Filho, Alvaro. II. Pereira, Mario Veiga Ferraz. III. Granville, Sergio. IV. Pontifícia Universidade Católica do Rio de Janeiro. Departamento de Engenharia Elétrica. V. Título. 
Para meus pais, Décio e Arlete com afeto, gratidão e saudade.

Para você, Nena com todo amor. 


\section{Agradecimentos}

Ao meu orientador Alvaro Veiga $\mathrm{F}^{\mathrm{o}}$ pelo estímulo e apoio.

A FURNAS, ao CNPq e à PUC-Rio pelos auxílios concedidos, sem os quais este trabalho não poderia ter sido realizado.

Aos professores que participaram da comissão julgadora.

Aos amigos Ajax R. B. Moreira e Katia Rocha do IPEA, pelo desenvolvimento das idéias, pela iluminação das dúvidas, pela motivação e pelo apoio absolutamente fundamentais.

Aos amigos e orientadores Mario Veiga Ferraz Pereira e Sergio Granville, fundamentais e cuja generosidade só rivaliza com a inteligência e conhecimento.

Aos diretores, superintendentes e gerentes de FURNAS, Celso Ferreira e Fabio Resende, Adelino Ferreira, Edmundo Pochmann e Luiz Roberto Alves Correa, Carlos Eduardo de Almeida Silva, Silvia Maria de Carvalho Celestino e Claudia de Barros Cotia, pelo apoio e incentivo; e aos meus colegas de FURNAS, em especial a Luiz Laércio S. Machado Jr., pelas discussões incentivadoras.

Aos professores da PUC-Rio, em especial aos Prof ${ }^{\mathrm{s}}$. Reinaldo Castro, Mônica Barros, Tara Baidya, Carlos P. Samanez e Love Ekenberg (Stockholm University) e aos funcionários do DEE, em especial à Alcina; à University of British Columbia - Faculty of Commerce, em

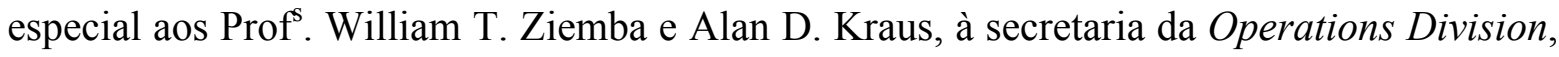
Betty Gelean e aos colegas, em especial, a Issouf Soumaré.

Aos meus amigos da PSR Consultoria, em especial à Luiz Augusto N. Barroso e Rafael Kelman; aos amigos da PUC-Rio, em especial, à Alessandro Castro e Leonardo L. Gomes; e do ONS, em especial à Alberto Kligerman e Mirtis Couto.

Às minhas irmãs, Analucia e Claudia e aos amigos Carlos Alberto da Silva Neto, Jorge Jardim, Mauro Muniz, Rita Rodrigues, Rogério Rocha e Salvatore Mantuano, pelo afeto, apoio e incentivo.

À minha esposa e companheira, Nena, pelo amor e apoio incondicionais ao longo dessa caminhada. 


\section{Resumo}

David, Pedro A. M-S.; Veiga $\mathrm{F}^{\mathrm{o}}$, Alvaro. Formação do Preço, Atração de Investimentos e Gerenciamento de Risco no Mercado Brasileiro de Energia Elétrica. Rio de Janeiro, 2003, 134 p. Tese de Doutorado - Departamento de Engenharia Elétrica, Pontifícia Universidade Católica do Rio de Janeiro.

O mercado brasileiro de energia elétrica ainda não encontrou um modelo de mercado e de formação de preço que garanta a expansão auto-sustentada da oferta. Investigando em detalhe o modelo atual de despacho da geração e formação do preço, demonstramos a sua pouca eficácia na atração de investimentos, e identificamos a causa dessa falha como sendo a miopia do modelo de despacho, uma vez os estados críticos do sistema só aprecem de forma significativa quando o sistema já estiver degradado. São estudados três modelos alternativos que modificam a função-objetivo ou a regra de formação do preço, ajustados de modo a viabilizar e tornar suficientemente atrativos os investimentos na expansão da oferta. Finalmente, estes modelos são então comparados entre si e com o modelo atual, quanto ao valor para o investidor e quanto ao custo para o sistema e para o consumidor.

Um mercado é dito "completo" se permite aos agentes alocar livremente seus recursos e demandas quando estiverem disponíveis e/ou forem necessários e permite que os agentes condicionem estes recursos / demandas ao estado (preço) do mercado. Estas funcionalidades são implementadas através dos derivativos financeiros, negociados no "mercado futuro". Neste trabalho fazemos uma análise conceitual do mercado futuro de energia elétrica, indicando a diferença em relação ao de outras commodities e apresentando um modelo da oferta e demanda por contratos futuros de energia elétrica.

\section{Palavras-chave}

Engenharia Elétrica; Mercado de Energia Elétrica; Modelos de Mercado e Formação do Preço; Viabilidade de Investimentos em Geração; Contratos Futuros de Energia Elétrica. 


\section{Abstract}

David, Pedro A. M-S; Veiga $\mathrm{F}^{\mathrm{o}}$, Alvaro (Advisor). Spot Price Formation, Investment Attraction and Risk Management in the Brazilian Electrical Energy Market. Rio de Janeiro, 2003, 134 p. Doctorate Thesis - Electrical Engineering Dept., Pontifícia Universidade Católica do Rio de Janeiro.

The Brazilian Market of Electrical Energy has not yet found a stable market and price model that ensues the feasibility and makes attractive a self-sustained investment for the expansion of electrical energy generation.

Researching the current generation dispatch and spot price model, we show that it is ineffective to attract investments because the model is myopic, since the range of critical system states that is foreseen at the current state is not significant until the system is already too degraded. Stemming from this conclusion, we develop three alternative models, modifying the dispatch model objective and the price formation rule. These alternative models are tuned to make the investments in generation expansion feasible and attractive. The models are compared regarding their value to the investor and the cost to the system and to the consumer.

A complete market allows the economic agents to freely allocate their resources and requirements whenever they are available and/or required. A complete market also allows conditional settlement, i.e., to condition the resource availability and/or requirement to a particular market state (price). These features are realized by financial derivatives, in the, so called, "futures market". We present a conceptual analysis of the electrical energy's future market, pointing the differences to other commodities' future markets that are due to economical unfeasibility of storing electricity. We also present an "equilibrium model" for the forward electrical energy contracts.

\section{Keywords}

Electrical Engineering; Electrical Energy Market; Market and Spot Price Models; Feasibility of Investments in Power Generation; Electrical Energy Forward Contracts. 


\section{Sumário}

1 Introdução 14

1.1 Resumo da História Econômica do Sistema Elétrico Brasileiro 15

1.2 Formação do Preço e Expansão da Oferta 18

1.3 Mercado Futuro de Energia Elétrica 20

$\begin{array}{lll}1.4 & \text { Revisão Bibliográfica } & 21\end{array}$

1.5 Estrutura da Monografia 24

$2 \quad$ Mercado de Energia Elétrica 25

2.1 Características Econômicas da Energia Elétrica 26

2.2 Mercado Brasileiro de Energia Elétrica 34

3 Decisão sob Incerteza $\quad 41$

3.1 Introdução 42

3.2 Utilidade Esperada 43

3.3 Dominância Estocástica 54

3.4 Heurísticas de Decisão sob Incerteza Absoluta 61

4 Formação do Preço e Expansão da Oferta 63

4.1 Introdução $\quad 64$

4.2 Modelo de Despacho da Geração Hidrotérmica 65

4.3 Modelos Alternativos de Despacho da Geração e Formação do Preço Spot74

4.4 Viabilidade Econômica da Expansão da Oferta 82

4.5 Estudo de Caso 86

$5 \quad$ Mercado Futuro de Energia Elétrica 103

5.1 Introdução 104

5.2 Mercado Futuro de Commodities 106

5.3 Oferta e Procura por Contratos de Energia 109

6 Conclusão 125

$\begin{array}{ll}6.1 & \text { Principais Resultados } \\ \end{array}$ 
6.2 Sugestões de Pesquisa Futura

$7 \quad$ Bibliografia 


\section{Lista de figuras}

Figura 1 - Sistema Brasileiro de Geração Hidrelétrica

Figura 2 - Distribuição Marginal da Potência Instalada das Usinas Hidrelétricas35

Figura 3 - Sistema Elétrico Brasileiro

Figura 4 - Incerteza do Preço Spot no Longo Prazo

Figura 5 - Energia Natural Afluente (Séries Históricas - Sudeste - 2004) 37

Figura 6 - Rateio da Produção Hidrelétrica (MRE) 39

Figura 7 - Função Utilidade - Neutralidade ao Risco 47

Figura 8 - Função Utilidade - Aversão ao Risco 48

Figura 9 - Função Utilidade - Atração pelo Risco 48

Figura 10 - Curvatura da Função Utilidade x Aversão ao Risco 49

Figura 11 - Dominância Estocástica de $1^{\text {a }}$. Ordem 56

Figura 12 - Dominância Estocástica de 2ª . Ordem 57

Figura 13 - Fronteira Eficiente e Dominância Estocástica 59

Figura 14 - Dominância Estocástica de 3a. Ordem 59

Figura 15 - Cálculo Recursivo do Custo Operativo 66

Figura 16- Custo Marginal da Geração Térmica e do Déficit 67

Figura 17 - Aproximação Linear por Partes de uma Função Convexa 69

Figura 18 - Equilíbrio de Mercado a Longo Prazo 70

Figura 19 - Déficit de Energia x Custo de Déficit 71

Figura 20 - CMO x Custo de Déficit $\quad 72$

Figura 21 - Custo Operativo em função do Estoque e da Afluência 72

Figura 22 - Distribuição Produção Hidrelétrica Modelos Original e Avesso a Risco 76

Figura 23 - Estoque de Água nos Reservatórios Modelos Original e Avesso a Risco 77

Figura 24 - Distribuição P. Spot Modelos Original e com Aversão a Risco 77

Figura 25 - Distribuição P. Spot - Modelos Original e com Preço Mínimo 79

Figura 26 - Distribuição P. Spot - Modelos Original e com Margem Mínima 80 
Figura 27 - Despacho da Geração Hidrelétrica - Modelos Original e com Margem Mínima 81

Figura 28 - Estoque de Energia - Modelos Original e com Margem Mínima 81

Figura 29 - Processo de Decisão de Investimento com Opção de Espera 82

Figura 30 - Valor da Opção de Compra x Incerteza dos Resultados 83

Figura 31 - Evolução da Capacidade de Geração 87

Figura 32 - Rentabilidade do Investimento em Geração Hidrelétrica Modelo com Aversão ao Risco

91

Figura 33 - Rentabilidade do Investimento em Geração Hidrelétrica Modelo com Margem de Investimento

Figura 34 - Rentabilidade do Investimento em Geração Hidrelétrica Modelo com Preço Mínimo

92

Figura 35 - Rentabilidade do Investimento em Geração Hidrelétrica Todos os Modelos 92

Figura 36 - Rentabilidade do Investimento em Geração Termelétrica Modelo com Aversão ao Risco

94

Figura 37 - Rentabilidade do Investimento em Geração Termelétrica Modelo com Margem de Investimento

Figura 38 - Rentabilidade do Investimento em Geração Termelétrica Modelo com Preço Mínimo

Figura 39 - Rentabilidade do Investimento em Geração Termelétrica Todos os Modelos 95

Figura 40 - Rentabilidade do Investimento no Portfolio de Geração Hidrotérmica Modelo com Aversão ao Risco

97

Figura 41 - Rentabilidade do Investimento no Portfolio de Geração Hidrotérmica Modelo com Margem de Investimento

97

Figura 42 - Rentabilidade do Investimento no Portfolio de Geração Hidrotérmica Modelo com Preço Mínimo

98

Figura 43 - Rentabilidade do Investimento no Portfolio de Geração Hidrotérmica Todos os Modelos 98

Figura 44- Reprodução de um Contrato Futuro através de Contratos de Opção105

Figura 45 - Grau de Aversão Risco e Portfolio de Geração 
Figura 46 - Curva de Oferta e Demanda por Contratos pelo Produtor $-(\lambda=6.791)$

Figura 47 - Curva de Oferta e Demanda por Contratos pelo Produtor $-(\lambda=24.160)$ 116

Figura 48 - Curva de Oferta e Demanda por Contratos pelo Produtor - $(\lambda=49.642)$ 116

Figura 49 - Curva de Oferta e Demanda por Contratos pelo Consumidor $-\lambda=6.791$ 119

Figura 50 - Curva de Oferta e Demanda por Contratos pelo Consumidor - $\lambda=24.160$ 120

Figura 51 - Curva de Oferta e Demanda por Contratos pelo Consumidor - $\lambda=49.642$ 120

Figura 52 - Oferta e Procura por Contratos 121

Figura 53 - Preço de Equilíbrio x Aversão ao Risco 122

Figura 54 - Nível de Contratação x Aversão ao Risco 123

Figura 55 - Elasticidade Preço do Produtor 124

Figura 56 - Elasticidade Preço do Consumidor 124 
Where there is a wish, there is a way William Shakespeare 